\title{
REDES ACADÉMICAS FRACTALES DE LA UNIVERSIDAD NACIONAL ABIERTA Y A DISTANCIA
}

\author{
Gloria C. Herrera Sáchez, ${ }^{63}$ Campo Elías Riaño Luna ${ }^{64}$
}

\begin{abstract}
RESUMEN
La UNESCO (1999) declara que el aprendizaje abierto y a distancia y el uso de las TIC's ofrecen oportunidades de ampliar el acceso a la educación de calidad, en particular cuando los recursos educativos abiertos son compartidos fácilmente por varios países y establecimientos de enseñanza superior, reconociéndose así su importancia y misión.

En las universidades a distancia esta revolución no sólo ha dependido del uso de las TIC's, también ha significado el desarrollo de otras iniciativas institucionales, con el fin de garantizar las condiciones de calidad, sostenibilidad, acceso, equidad y competitividad.

En este contexto, el presente artículo tiene como propósito, mostrar los logros académicos (docencia, investigación, proyección social, administración y consejería) de la Universidad Nacional Abierta y a Distancia de Colombia (UNAD), como resultado de su transformación organizacional y pedagógica, iniciada en el 2006 y centrada en el desarrollo de una estructura sistémica, reticular, fractal y heterárquica; lo que ha favorecido el surgimiento y sostenimiento de redes académicas y la consistencia de una comunidad educativa a distancia con proyección internacional.
\end{abstract}

Palabras clave: redes, redes académicas, reticularidad, fractalidad, heterarquia.

\begin{abstract}
The UNESCO (1999) declares that the opened learning and distantly and the use of the TIC's they offer opportunities to extend the access to the quality education, especially when the educational opened resources are shared easily between several countries and establishments of higher education, his importance and mission being recognized like that.

In the universities distantly this revolution not only has depended on the use of the TIC's, also it has meant the development of other institutional initiatives, in order to guarantee the conditions of quality, sustainability, access, equity and competitiveness.

In this context, this one article has as intention, demonstrate the academic achievements (teaching, investigation, social projection, administration and Council) of the National Opened University and distantly of Colombia (UNAD), as result of his transformation organizacional

63 Vicerrectora Académica y de Investigaciones unAD. Estudiante Doctorado Tecnología Instruccional y Educación a Distancia. E-mail: gherrera@nova.edu.

64 Docente ECBTI. Estudiante Doctorado Tecnología Instruccional y Educación a Distancia. rianoluna@nova.edu.
\end{abstract}


and pedagogic, initiated in 2006 and centred on the development of a systemic, reticulated, fractal organization and heterarquica; what has favored the emergence and maintenance of academic networks and the consistency of an educational community distantly with international projection.

Key words: networks, academic networks, reticularidad, fractalidad, heterarquia

Recibido: 14 de abril de 2010

Aceptado: 23 de abril de 2010

\section{INTRODUCCIÓN}

En Colombia existen 282 instituciones de educación superior, pero la UNAD, desde su fundación en el año de 1981, ha sido única en el país que por su naturaleza, desarrolla el $100 \%$ de su servicio educativo en la modalidad de educación a distancia (Ministerio de Educación Nacional, 2009:9).

En el año de 1995 la Oficina de Registro y Control Académico de la UNAD, reporta un promedio de diez mil setecientos veintitrés (10.723) estudiantes matriculados en los semestres 1 y 2 . En el 2004 matriculó en promedio veintisiete mil doscientos ochenta y dos (27.282) estudiantes, cifra que indicaba que la cobertura había tenido un incremento de $254 \%$ (25.4\% promedio anual). El personal administrativo y académico para este mismo año a nivel nacional era de dos mil ciento cuarenta y nueve (2.149) funcionarios, lo cual dificultaba la prestación de un servicio oportuno y pertinente con las características de los usuarios, de la época, de la modalidad de educación ofrecida y del país.

La reflexión sobre el comportamiento de la matrícula, la calidad del servicio educativo, el desempeño administrativo y eficacia de los programas académicos, así como la prospectiva de la educación a distancia, llevó a la comunidad universitaria a desarrollar un proceso de planeación estratégica situacional, empleando el método Altadir de Planificación Popular (MAPP), como se propone en el Fondo Editorial Altadir (1994).

En esta comisión se declaró como misión de la UNAD (2006b), "Contribuir a la educación para todos a través de la modalidad abierta y a distancia, mediante la investigación, la acción pedagógica, la proyección social y las innovaciones metodológicas y didácticas, con la utilización de las TIC's, para fomentar y acompañar el aprendizaje autónomo, generador de cultura y espíritu emprendedor que en el marco de la sociedad global y del conocimiento propicie el desarrollo económico, social y humano sostenible de las comunidades locales, regionales y globales con calidad, eficiencia y equidad social". Para el efecto se propuso la constitución de un sistema nacional de redes, orientadas desde nodos nacionales e internacionales, enlazando centros satélites de educación a distancia. De esta forma, se prepara para exportar servicios, conocimientos y experiencias hacia otras latitudes y compromete a la comunidad educativa con la transformación de la organización y el modelo pedagógico; así como con la ampliación de cobertura, el afianzamiento de las redes de conocimiento, de la 
pedagogía mediada a través de las TIC's y el fortalecimiento del arraigo regional. Conjunto de acciones que permitieron desarrollar condiciones propias de una organización sistémica, reticular, fractal y heterárquica, ver gráfica 1.

Gráfica 1. Estructura organizacional de la UNAD

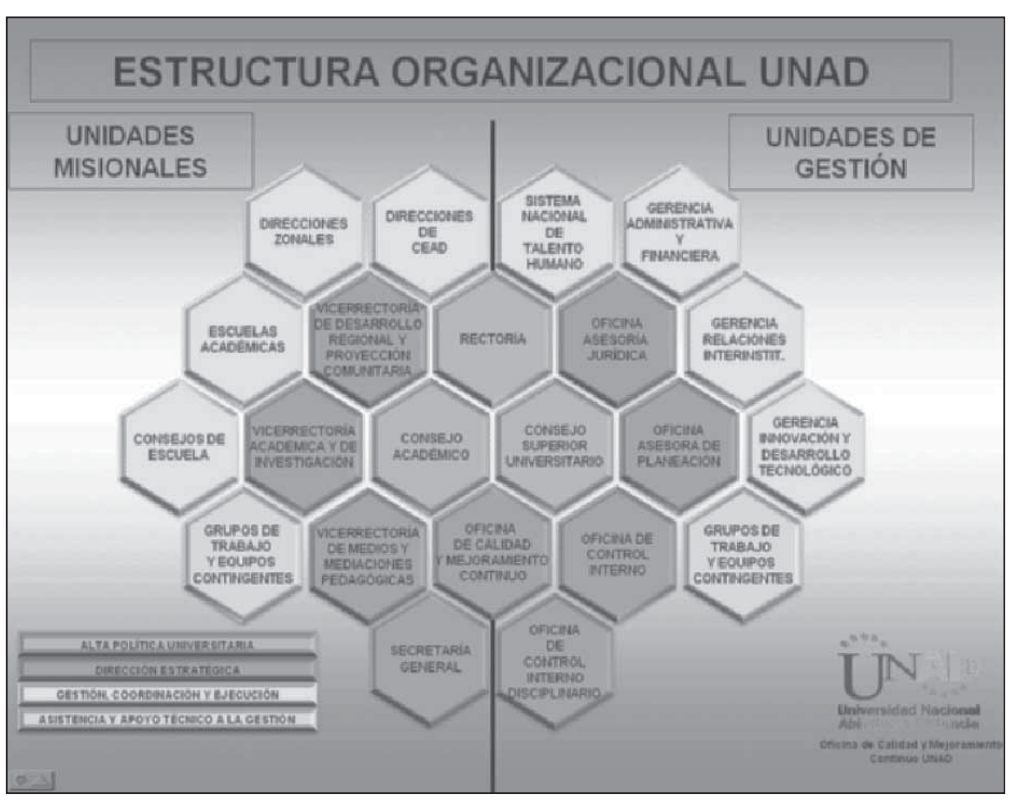

Fuente: UNAD (2006a)

\section{Pregunta de Investigación}

Para desarrollar el presente trabajo partimos de la pregunta de investigación:

¿Cómo la UNAD, se convierte en una organización inteligente que aprende de la fractalidad, de la reticularidad, de la heterarquia y del trabajo en red?

\section{Necesidades de las Redes Académico- Administrativas en la UNAD}

La UnAD(2006b:3) en el estatuto General, enuncia como objetivos misionales los siguientes: “c) Promover la construcción de redes apoyadas en tecnologías, para consolidar las comunidades académicas de docentes, tutores, estudiantes y egresados, que promuevan los principios y las prácticas de la identidad Unadista, y generen nuevas formas de asociación, convivencia y participación comunitaria".

La alta dispersión geográfica de los estudiantes, habitantes de 907 de los 101 municipios del país, llevó a la universidad a crear estrategias de presencia regional y local: 39 Centros 
de Educación a Distancia (CEAD) localizados en ciudades grandes y medianas, 17 Centros Regionales de Educación Superior (CERES) ubicados en municipios pequeños, 2 Centros Comunitarios de Atención Virtual (CCAV), 2 Unidades de Desarrollo Regional (UDR) localizados en comunidades con alta presencia rural, 2 Centros de Frontera, 1 Centro Internacional (UNAD Florida - USA), como se muestra en la gráfica n. ${ }^{\circ} 2$. Razón por la cual la UNAD, creó un campus virtual con el propósito de facilitar los procesos académicos, atender a los usuarios y desarrollar innovación pedagógica, trascendiendo de una educación a distancia con encuentros presénciales periódicos entre los estudiantes y los docentes en los 63 centros dispuestos en el territorio colombiano, a una educación a distancia e-Learning, funcionando con el principio de redes de alta velocidad establecidas entre entidades académicas de la UNAD, dedicadas exclusivamente a la investigación, al desarrollo educativo, la atención de usuarios y a los procesos administrativos, lo cual implica trabajar con procesos académico-educativos virtuales, proyectos de colaboración en tiempo real utilizando video y audio conferencias. Esta innovación dio como resultado el desarrollo de condiciones propias de una organización sistémica, reticular, fractal y heterárquica. La reticularidad en la UNAD beneficia el desarrollo de las actividades académicas y administrativas, el trabajo en equipo y la constitución de comunidad educativa y, la fractalidad favorece el desarrollo de la identidad y presencia institucional en contextos locales, regionales e internacionales. Permitiendo que los subsistemas funcionales de la UNAD, interactúen con las redes conformadas por usuarios, por grupos de trabajo, centros de atención, dependencias y estamentos superiores de alta política universitaria; integrando comunidades administrativas, educativas, investigativas e informativas, y reconocidas como un todo o desde cada una de sus partes. Ratificándose lo expresado por Mandelbrot y Hudson (2006:17) y Gálvez (2005:23) cuando definen a un fractal como "una forma geométrica que puede fraccionarse en partes menores, cada una de las cuales evoca la totalidad a menor escala, lo que significa que tiene propiedades como la auto-similitud y auto-semejanza a pesar de la irregularidad, condiciones que contribuyen a la alineación de una comunidad académica a pesar de la dispersión geográfica de sus integrantes".

Igualmente la presentación, el desarrollo, la adopción y aplicación de las actividades emprendidas para la transformación sistémica, organizacional, pedagógica y estructural de la UNAD, han sido presentadas a la comunidad con el menor grado de complejidad posible, a fin de facilitar la tasa de adopción, como lo propone Rogers (2003). Requisito que se cumplió puesto que se han generado siete grandes (7) redes de actuación. 
Gráfica 2. Trabajo colaborativo en red en las zonas de influencia de la UNAD

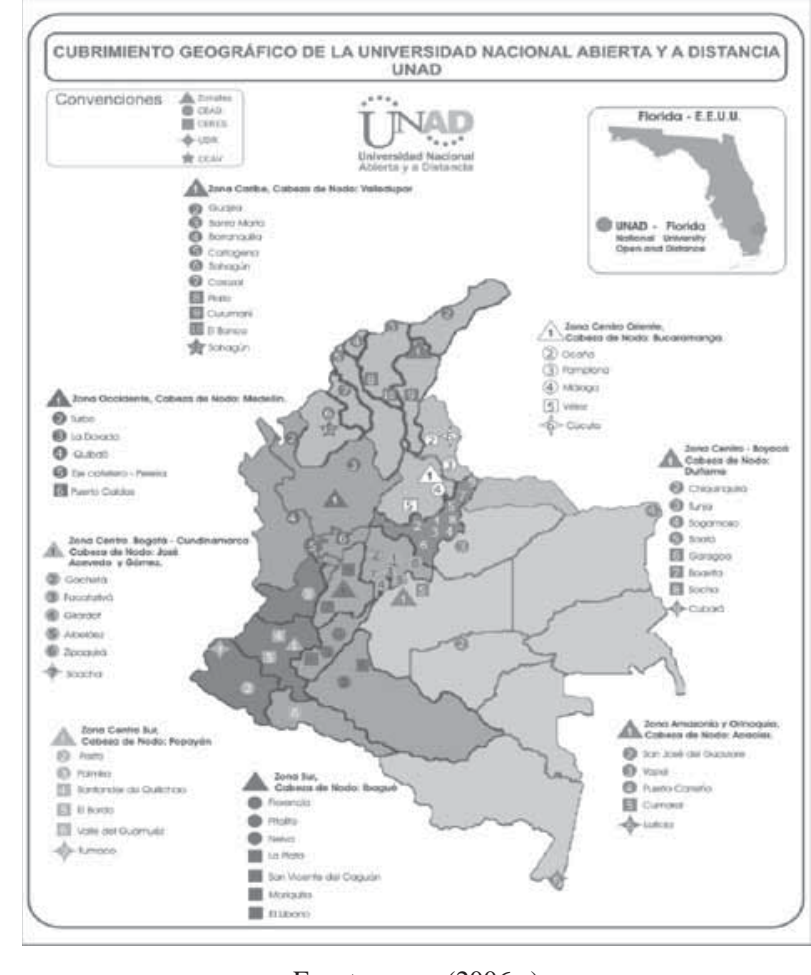

Fuente: UNAD (2006 c)

\section{Redes Académicas en la UNAD}

Las redes en la institución tienen la misión de compartir los recursos de las Tecnologías de la Información y la Comunicación (TIC), mediante procesos de trabajo conjunto o complementario entre directivos, coordinadores, el cuerpo académicos y todos los integrantes de la comunidad unadista. Para el efecto se desarrollan escenarios donde estudiantes, tutores, docentes, investigadores, administrativos, directivos y egresados con un alto grado de autonomía, heterogéneos en su forma de pensar y de hacer, y distantes geográficamente, aúnen esfuerzos en torno a propósitos comunes. Para el efecto cada una de las siete redes tiene objetivos generales (tabla 1), comparte recursos y esfuerzos y transita de una visión de cadena informativa- productiva, a una de redes colaborativas- informativas-productivas. 
Tabla 1. Redes de la unAD, objetivos y logros

\begin{tabular}{|c|c|c|}
\hline Red & Objetivo general & Identificación \\
\hline Docentes y tutores & $\begin{array}{l}\text { Propiciar el encuentro, la } \\
\text { interacción y la construcción } \\
\text { de conocimiento en el campo } \\
\text { de la pedagogia virtual }\end{array}$ & ZibyncaRed \\
\hline $\begin{array}{l}\text { Investigación y gestión de } \\
\text { conocimiento }\end{array}$ & $\begin{array}{l}\text { Integrar a los grupos, } \\
\text { semilleros, investigadores y } \\
\text { estudiantes, brindándoles un } \\
\text { espacio de convergencia. }\end{array}$ & 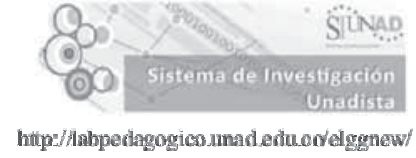 \\
\hline Proyección social & $\begin{array}{l}\text { Generar espacios para la } \\
\text { interaceión social, la } \\
\text { convivivencia y la conectividad. } \\
\text { Definida fundamentalnente } \\
\text { por los intercambios dinámicos } \\
\text { entre las instituciones que la } \\
\text { forman. }\end{array}$ & 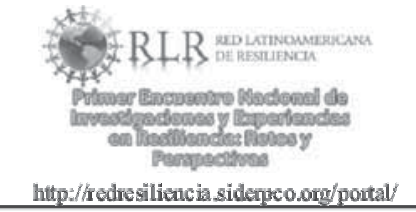 \\
\hline $\begin{array}{l}\text { Producción de materiales } \\
\text { didácticos. } \\
\text { Red de OVAS }\end{array}$ & $\begin{array}{l}\text { Construir un espacio de } \\
\text { intercomunicación y de trabajo } \\
\text { colaborativo, que facilite la } \\
\text { construcéón y utilización de } \\
\text { objetos virtuales de aprendizaje } \\
\text { como estrategia pedagógica y } \\
\text { didáctica multimedial a través } \\
\text { de los cursos académicos. }\end{array}$ & $\begin{array}{r}\text { Red de } \\
\text { OVAS }\end{array}$ \\
\hline Consejería académica & $\begin{array}{l}\text { Asesorar y contribuir con el } \\
\text { desaurollo formativo del } \\
\text { estudiante. }\end{array}$ & $\frac{6}{212}$ \\
\hline $\begin{array}{l}\text { Sistema Nacional de } \\
\text { Registro y Control } \\
\text { Acadénnico }\end{array}$ & $\begin{array}{l}\text { Generar información sobre los } \\
\text { procesos acadéninicos. }\end{array}$ & $\begin{array}{c}\text { Registro y } \\
\text { Control }\end{array}$ \\
\hline $\begin{array}{l}\text { Coordinadores misionales } \\
\text { académicos zonales }\end{array}$ & $\begin{array}{l}\text { Difundir y promocionar } \\
\text { actividades y experiencias } \\
\text { docentes y de investigación en } \\
\text { las zonas y a nivel nacional. }\end{array}$ & \\
\hline
\end{tabular}

Fuente: UNAD

\section{Crecimiento de las Redes en la UNAD}

De la teoría de la difusión de Rogers (2003) (como se cita en Simonson 2006) y de Salinas (1998, 1.999a, 1999b), retomamos el concepto de masa crítica (tiempo requerido por cierto porcentaje de miembros de un sistema social para adoptar una innovación), y encontramos el comportamiento progresivo de las seis redes reportadas en la tabla 2. 
Tabla 2. Redes Académicas UNAD 2004 -2009

\begin{tabular}{|c|r|r|r|r|r|r|}
\hline \multirow{2}{*}{ Año } & \multicolumn{6}{|l|}{ Orientación de la Red } \\
\cline { 2 - 7 } & $\begin{array}{c}\text { Docentes } \\
\text { y tutores }\end{array}$ & $\begin{array}{c}\text { Investigación } \\
\text { y gestión de } \\
\text { conocimiento }\end{array}$ & $\begin{array}{c}\text { Proyección } \\
\text { social }\end{array}$ & $\begin{array}{c}\text { Red de } \\
\text { OVAS }\end{array}$ & $\begin{array}{c}\text { Consejería } \\
\text { académica }\end{array}$ & $\begin{array}{c}\text { Registro y } \\
\text { control }\end{array}$ \\
\hline 2006 & 35 & 0 & 0 & 1 & 8 & 2 \\
\hline 2007 & 191 & 0 & 0 & 3 & 8 & 4 \\
\hline 2008 & 335 & 10 & 5 & 6 & 9 & 6 \\
\hline 2009 & 401 & 15 & 13 & 14 & 13 & 10 \\
\hline
\end{tabular}

En donde se ve que cada red ha tenido un crecimiento ligeramente diferente. No hay un modelo único. En unas existen muchos usuarios como la red de docentes y tutores, en otras el número de miembros es menor. La red de registro y control llego a su número máximo de nodos (10).

\section{Realizaciones de las Redes en la UNAD}

Las redes en la UNAD tienen como características importantes:

a-Su carácter colaborativo en línea. Ejemplo es el sistema nacional de registro y control operando en red con los nodos, en la tabla 3, se corrobora el esfuerzo institucional por extender la cobertura educativa en el país, la cual en los últimos cinco años ha tenido un incremento de la matrícula en línea del 182\% (promedio anual de 36.4\%), superando el crecimiento proyectado. Informe de Estadísticas de la Educación Superior (2002): "2009 (30.70\%), 2007 (31.80\%), 2008 (33\%)" (p. 2).

Tabla 3. Incremento en la matrícula UNAD 2004 - 2009

\begin{tabular}{|c|c|c|}
\hline Año & Población matriculada & Incremento ( 2 periodos por año) \\
\hline 2004-I & 28.185 & \multirow{2}{*}{$26.9 \%$} \\
\hline 2004-II & 26.378 & \\
\hline 2005-I & 27.944 & \multirow{2}{*}{$28.9 \%$} \\
\hline 2005-II & 29.227 & \\
\hline 2006-I & 32.226 & \multirow{2}{*}{$30.6 \%$} \\
\hline 2006-II & 33.462 & \\
\hline 2007-I & 37.224 & \multirow{2}{*}{$31.8 \%$} \\
\hline 2007-II & 46.628 & \\
\hline 2008-I & 44.530 & \multirow{2}{*}{$34.1 \%$} \\
\hline 2008-II & 44.697 & \\
\hline 2009-I & 49.433 & \multirow{2}{*}{$35.5 \%$} \\
\hline 2009-II & 50.300 & \\
\hline
\end{tabular}

Fuente: UNAD (2009a) Sistema Nacional de Registro y Control Académico 
b-Su accionar a partir de los principios de actuación fractal y su capacidad de replicarse en subredes. Ejemplo es la red del sistema nacional de información de investigaciónSIUNAD, estamento que anualmente realiza dos convocatorias; para financiar proyectos de investigación. Proceso llevado a cabo completamente en línea, con participación de los nodos y de la comunidad académica, sus resultados se encuentran en la grfiáca 3.

Gráfica 3. Distribución de proyectos de investigación por redes temáticas

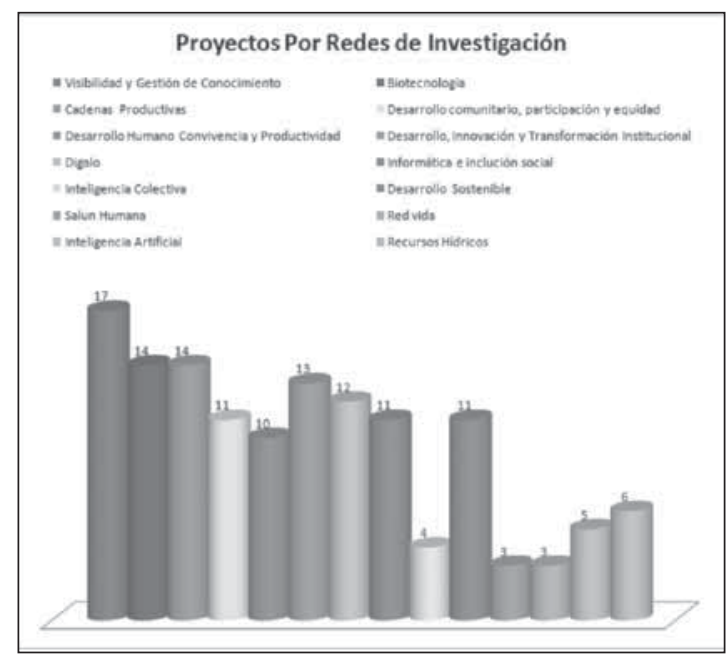

Fuente: Informes de Gestión 2009

c-Y su capacidad de auto-organizarse de manera independiente, siempre que sean coherente con los principios y valores de la institución. La experiencia que la UNAD ha venido acumulando en este aspecto se reporta en la tabla 4.

Tabla 4. Auto- realización de las Redes Académicas

\begin{tabular}{|l|c|l|}
\hline \multicolumn{1}{|c|}{ Redes } & Nodos proactivos & \multicolumn{1}{c|}{ Subredes } \\
\hline Red de investigación & 15 & $\begin{array}{l}\text { 99 grupos de investigación avalados } \\
39 \text { semilleros de investigación } \\
141 \text { proyectos de investigación }\end{array}$ \\
\hline Redes de consejeros & 13 & 13 (asocian 89 consejeros) \\
\hline Redes de tutores de curso & 401 & 491 (asocian 1588 tutores) \\
\hline Redes de proyección social & 13 & $\begin{array}{l}\text { Líneas de acción de la Vicerrectoría de } \\
\text { Desarrollo Regional y proyectos específicos }\end{array}$ \\
\hline $\begin{array}{l}\text { Redes de producción de } \\
\text { materiales didácticos }\end{array}$ & 6 & $\begin{array}{l}\text { Líneas de acción de la Vicerrectoría de Medios y } \\
\text { Mediaciones Pedagógicas y proyectos específicos }\end{array}$ \\
\hline
\end{tabular}

Fuente: UNAD (2009a) 
En esta tabla (4) se demuestra la capacidad de la UNAD para construir escenarios de interacción en todos los niveles académicos-administrativos, y sobre todos los componentes que intervienen en la comunicación, la pedagogía mediada a través de las TIC's y la promoción, uso y construcción de mediaciones pedagógicas interactivas.

\section{CONCLUSIONES}

Los resultados de este ejercicio, muestran el compromiso y la laboriosidad de la comunidad educativa de la UNAD para el trabajo colaborativo en red, la transformación de la organización y del modelo pedagógico, la ampliación de la cobertura geográfica y poblacional en beneficio del servicio educativo para el País, el afianzamiento de las redes de conocimiento, el fortalecimiento del arraigo regional y la transmisión de experiencias docentes y de resultados de investigación.

Y finalmente la réplica de las redes en subredes demuestra la capacidad para construir, prospectar, anticipar y reaccionar a la velocidad del cambio, lo que puede asumirse como acción propia de la inteligencia organizacional.

\section{REFERENCIAS BIBLIOGRÁFICAS}

FONDO EDITORIAL ALTADIR. (1994). Método MAPP. Método Altadir de Planificación Popular. Bogotá: Colección Ciencias y Técnicas de Gobierno.

GÁLVEZ, E. (2005). Análisis fractal del mercado de valores de México (1978-2004).México: Instituto Politécnico Nacional.

MANDELBROT, B., \& HUDSON, R. (2006). Fractales y finanzas. Barcelona: Tusquets Editores S.A.

MINISTERIO DE EDUCACIÓN NACIONAL. (2002). Estadísticas de la Educación Superior 2002. Consultado Noviembre 18 de 2009 en http://menweb.mineducacion.gov. co/info_sector/estadisticas/index

MINISTERIO DE EDUCACIÓN NACIONAL. (2009). Observatorio de la Universidad Colombiana: Cobertura de la educación superior en Colombia. Consultado el 18 de Noviembre de 2009 en: http://www.universidad.edu.co

ROGERS, EVERETT. (2003). Diffusion of Innovations. 5a. editión. New York. Free Press.

SALINAS, J. (1999a). Uso educativo de las redes informáticas. Consultado en Noviembre 21 de 2009 en: www.edutec.rediris.es/documentos/1999/educar.html. 
ReVISTA DE INVEStigaciones UNAD

SALINAS, J. (1998). Redes y desarrollo profesional del docente: Entre el dato serendipiti y el foro de trabajo colaborativo. Rev. Profesorado (Univ. de Granada), 2 (1). Consultado en septiembre 18 de 2009 en: http://www.uib.es/depart/gte/docente.html

SALINAS, J. (1.999b). Rol del profesorado universitario ante los cambios de la era digital. Perfeccionamiento Integral del Profesor Universitario, Primer Encuentro Iberoamericano. Universidad Central de Venezuela. Caracas. Julio 1.999-10-18.

SIMONSON, M., SMALDINO, S., ALBRIGHT, M., \& ZVACEK. S. (2006). Teaching and learning at a distance: Foundations of distance education,3ed. Upper Saddle, NJ: Prentice-Hall.

UNESCO (1999). Conferencia Mundial sobre la Educación Superior en el Siglo XXI: Visión y Acción. Consultado en Noviembre 18 de 2009 en: http://unesdoc.unesco.org/ images/0011/001170/117022S

UNIVERSIDAD NACIONAL ABIERTA Y A DISTANCIA. (2006a). Normatividad institucional. Bogotá: UNAD.

UNIVERSIDAD NACIONAL ABIERTA Y A DISTANCIA. (2006b). Estatuto General. Bogotá: UNAD.

UNIVERSIDAD NACIONALABIERTAYADISTANCIA. (2006c). Estatuto Organizacional. Bogotá: UNAD.

UNIVERSIDAD NACIONAL ABIERTA Y A DISTANCIA. (2009a). Informe de Gestión 2009. Bogotá: UNAD.

UNIVERSIDAD NACIONAL ABIERTA Y A DISTANCIA. (2009b). Informe de gestión del sistema de investigación unadista-SIUNAD; análisis estadístico. Consultado el 28 de noviembre de 2009 de: http://labpedagogico.unad.edu.co/siunad/images/stories/ informesfinales/estadisticas2008-2009.pdf. 